TRANSACTIONS OF THE

AMERICAN MATHEMATICAL SOCIETY

Volume 356, Number 1, Pages 159-184

S 0002-9947(03)03037-X

Article electronically published on August 26, 2003

\title{
CLOSED PRODUCT FORMULAS FOR EXTENSIONS OF GENERALIZED VERMA MODULES
}

\author{
RICCARDO BIAGIOLI
}

\begin{abstract}
We give explicit combinatorial product formulas for the polynomials encoding the dimensions of the spaces of extensions of $(g, p)$-generalized Verma modules, in the cases when $(g, p)$ corresponds to an indecomposable classic Hermitian symmetric pair. The formulas imply that these dimensions are combinatorial invariants. We also discuss how these polynomials, defined by Shelton, are related to the parabolic $R$-polynomials introduced by Deodhar.
\end{abstract}

\section{INTRODUCTION}

Let $g$ be a complex, semisimple Lie algebra with Cartan subalgebra $h$ and let $b$ be a Borel subalgebra of $g$ with nilradical $n$ and Levi component $h$. Let $p$ be a parabolic subalgebra of $g$ containing $b$ with nilradical $u \subseteq n$ and Levi component $m \supseteq h$. So we have Levi decompositions $b=h \oplus n$ and $p=m \oplus u$. The problem of computing the $u$-cohomology of irreducible highest weight $(g, p)$-modules is completely solved by the Kazhdan-Lusztig conjectures ([7]). On the other hand, the "Extension Problem", namely the problem of computing the $u$-cohomology of the $(g, p)$-generalized Verma modules, is as yet unsolved in general, and there are not even conjectures for the form of the answer. If $p=b$, the modules in question are the Verma modules (see [9]). There was a conjecture that solved the problem, known as the Gabber-Joseph conjecture (see, e.g., 14] or [8), but this was disproved by Boe in [2]. In the cases when $(g, p)$ corresponds to an indecomposable Hermitian symmetric pair (see, e.g., [9, §4]), Shelton gives a solution to this problem. His answer is in terms of a recursion relations for the polynomials encoding the dimensions of the spaces of extensions between generalized Verma modules [17.

In this paper we solve these recursion relations, in the cases when $(g, p)$ is an indecomposable classic Hermitian symmetric pair (see Table 1 at the end of $\S 2$ ). Our main results are explicit combinatorial product formulas for these polynomials. These formulas can be stated in two different ways, one in terms of Weyl group elements and one in terms of partitions or shifted partitions. Moreover, these formulas imply that these polynomials are combinatorial invariants.

The organization of the paper is as follows. In the next section we collect some definitions and results that are needed in the rest of the work. In $\S 3$ we study the case of the symmetric pair $\left(A_{n-1}, A_{i-1} \times A_{n-i-1}\right)$, in $\S 4$ the case of $\left(C_{n}, A_{n-1}\right)$, in $\S 5$ the case of $\left(D_{n}, A_{n-1}\right)$, and in $\S 6$ the remaining cases $\left(B_{n}, B_{n-1}\right)$ and $\left(D_{n}, D_{n-1}\right)$. In the first three cases we give two statements of these results, one in terms of

Received by the editors September 24, 2001 and, in revised form, February 11, 2002.

2000 Mathematics Subject Classification. Primary 17B10, 05E99; Secondary 22E47, 20 F55. 
permutations or signed permutations and one in terms of partitions or shifted partitions. In $\S 7$ we derive some consequences of our results and in particular we prove that these polynomials are combinatorial invariants. We also discuss how they are related to the parabolic $R$-polynomials introduced in [12, and the pleasant "symmetry" that follows with the Gabber-Joseph conjecture [14].

\section{Notation, DEFINITIONS, AND PRELIMINARIES}

In this section we give some definitions, notation and results that will be used in the rest of this work. We let $\mathbf{P}:=\{1,2,3, \ldots\}, \mathbf{N}:=\mathbf{P} \cup\{0\}$, and $\mathbf{Z}$ be the set of integers; for $a \in \mathbf{N}$ we let $[a]:=\{1,2, \ldots, a\}$ (where $[0]:=\emptyset$ ) and $[ \pm n]:=$ $[-n, n] \backslash\{0\}$. The cardinality of a set $A$ will be denoted by $|A|$. For any sequence $\left(a_{1}, \ldots, a_{n}\right) \in \mathbf{Z}^{n}$, we let $N_{1}\left(a_{1}, \ldots, a_{n}\right):=\left|\left\{i \in[n]: a_{i}<0\right\}\right|$. Let $S_{n}$ be the set of all bijections $\sigma:[n] \rightarrow[n]$. If $\sigma \in S_{n}$, then we write $\sigma=\sigma_{1} \ldots \sigma_{n}$ to mean that $\sigma(i)=\sigma_{i}$, for $i=1, \ldots, n$. If $\sigma \in S_{n}$, then we will also write $\sigma$ in disjoint cycle form (see, e.g., [18, p.17) and we will usually omit writing the 1-cycles of $\sigma$. For example, if $\sigma=64175823$, then we also write $\sigma=(2,4,7)(1,6,8,3)$. Given $\sigma, \tau \in S_{n}$ we let $\sigma \tau:=\sigma \circ \tau$ (composition of functions) so that, for example, $(1,2)(2,3)=(1,2,3)$. We follow Chapter 3 of [18] for poset notation and terminology. In particular, given a poset $(P, \leq)$ and $u, v \in P$ we let $[u, v]:=\{z \in P: u \leq z \leq v\}$ and call this an interval of $P$. If $P$ has a minimal element, denoted $\hat{0}$, then we call a subset of the form $[\hat{0}, u]$, for $u \in P$, a lower interval of $P$. Given any $Q \subseteq P$ we will always consider $Q$ as a poset with the partial ordering induced by $P$ and call $Q$ a subposet of $P$. We say that $z \in P$ is join-irreducible if it covers at most one element of $P$. Given two posets $P$ and $Q$ we write $P \cong Q$ to mean that they are isomorphic as posets.

We follow $\S 7.2$ of [19] for any undefined notation and terminology concerning partitions. By an (integer) partition we mean a sequence of positive integers $\lambda=$ $\left(\lambda_{1}, \ldots, \lambda_{k}\right)$ such that $\lambda_{1} \geq \lambda_{2} \geq \ldots \geq \lambda_{k}$ and we write $|\lambda|=\sum \lambda_{i}$. The number of parts of $\lambda$ is the length of $\lambda$, denoted $l(\lambda)$. We identify a partition $\lambda$ with its diagram,

$$
\left\{(i, j) \in \mathbf{P}^{2}: 1 \leq i \leq k, 1 \leq j \leq \lambda_{i}\right\}
$$

If we replace the dots $(i, j)$ by juxtaposed squares, we obtain the Young diagram of $\lambda$, and we draw it rotated counterclockwise by $\frac{\pi}{4}$ radians. So, for example, the diagram of $(5,4,2,1)$ is illustrated in Figure 1.

We call the elements of $\mathbf{P}^{2}$, and hence of $\lambda$, cells. Expressions such as "to the left of", or "directly above", always refer to these rotated diagrams. We denote by $\mathcal{P}$ the set of all integer partitions. For any $\mu, \lambda \in \mathcal{P}$ we define $\mu \subseteq \lambda$ if and only

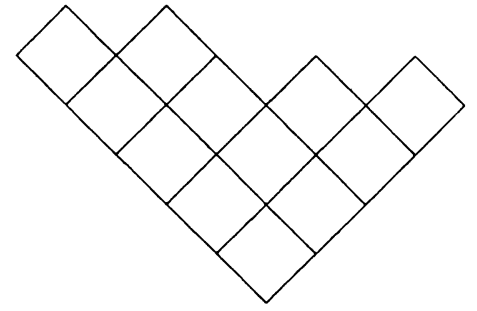

FiguRE 1. 
if $\mu_{i} \leq \lambda_{i}$ for all $i$. If we identify a partition with its (Young) diagram, then the partial order $\subseteq$ is given simply by containment of diagrams. It is well known, and not hard to see, that this makes $\mathcal{P}$ into a lattice, usually called Young's lattice (see, e.g., [19, §7.2]). Given $n \in \mathbf{P}$ and $i \in[n-1]$ we let

$$
\mathcal{P}(n, i):=\left\{\mu \in \mathcal{P}: \mu \subseteq(n-i)^{i}\right\} .
$$

Given $\lambda=\left(\lambda_{1}, \ldots, \lambda_{k}\right) \in \mathcal{P}$ we let $d(\lambda)$ be the length of the Durfee square of $\lambda$, so

$$
d(\lambda):=\max \left\{i \in[k]: \lambda_{i} \geq i\right\} .
$$

Let $\mu, \lambda \in \mathcal{P}, \mu \subseteq \lambda$. We then call $\lambda \backslash \mu$ a skew partition. Note that, in poset theoretic language, partitions (respectively, skew-partitions) are the finite order ideals (respectively, finite convex subsets) of $\mathbf{P}^{2}$. Given two skew partitions $\rho, \nu \subset \mathbf{P}^{2}$ we write $\rho \approx \nu$ if $\rho$ is a translate of $\nu$. Given a skew partition $\nu \subseteq \mathbf{P}^{2}$ its conjugate is

$$
\nu^{\prime}:=\left\{(j, i) \in \mathbf{P}^{2}:(i, j) \in \nu\right\} .
$$

We follow [15] for general Coxeter groups notation. In particular, given a Coxeter system $(W, S)$ and $\sigma \in W$ we denote by $l(\sigma)$ the length of $\sigma$ in $W$, with respect to $S$, and we let $D(\sigma):=\{s \in S: l(\sigma s)<l(\sigma)\}$. We denote by $e$ the identity of $W$, and we let $T:=\left\{\sigma s \sigma^{-1}: \sigma \in W, s \in S\right\}$ be the set of reflections of $W$. Given $J \subseteq S$ we let $W_{J}$ be the parabolic subgroup generated by $J$ and

$$
W^{J}:=\{\sigma \in W: l(s \sigma)>l(\sigma) \text { for all } s \in J\} .
$$

Note that $W^{\emptyset}=W$. The quotient $W^{J}$ is a poset and it is partially ordered by (strong) Bruhat order. Recall (see, e.g., [15, §5.9]) that this means that $x \leq y$ if and only if there exist $r \in \mathbf{N}$ and $t_{1}, \ldots, t_{r} \in T$ such that $t_{r} \ldots t_{1} x=y$ and $l\left(t_{i} \ldots t_{1} x\right)>l\left(t_{i-1} \ldots t_{1} x\right)$ for $i=1, \ldots, r$. In this order, $e$ is the minimal element and, if $W$ is finite, $w_{0}^{J}$ and $w_{J}^{0}$ denote the unique maximal elements in $W^{J}$ and $W_{J}$, respectively. Given $u, v \in W^{J}, u \leq v$, we let

$$
[u, v]^{J}:=\left\{z \in W^{J}: u \leq z \leq v\right\}
$$

and consider $[u, v]^{J}$ as a poset with the partial ordering induced by $W^{J}$.

In the remainder of this section, we recall a result of Shelton, for which we need some notation from [13] and [17. Let $g$ be a complex, semisimple Lie algebra with Cartan subalgebra $h$, and let $b$ be a Borel subalgebra of $g$ with nilradical $n$ and Levi component $h$. Let $p$ be a parabolic subalgebra of $g$ with nilradical $u$ and Levi component $m$. Let $W$ and $W_{J}$ be the Weyl groups of $g$ and $m$, respectively. Let $\Delta, \Delta(m)$ be the root systems of $g$ and $m$, with positive systems $\Delta^{+}$and $\Delta^{+}(m)$, determined by $n$. Let $2 \rho$ be the sum of the positive roots in $g$ and fix a $\Delta^{+}$-antidominant integral weight $\lambda \in h^{*}$. For any $\mu \in h^{*}$ we denote by $M(\mu)$ the $g$-Verma module with highest weight $\mu-\rho$. Then $L(\mu)$ and $N(\mu)$ denote the unique irreducible quotient and the maximal $m$-locally finite quotient of $M(\mu)$, respectively. If $\mu$ is $\Delta^{+}(m)$-dominant regular and integral, then $N(\mu)$ is the $(g, p)$ generalized Verma module of highest weight $\mu-\rho$. For $u \in W$ and $w \in W^{J}$ we denote by $M_{u}$ the module $M(u \lambda)$ and by $N_{w}$ the module $N\left(w_{J}^{0} w \lambda\right)$. All Ext* groups will be computed in $\mathcal{O}_{m}$, the usual Bernstein-Gelfand-Gelfand category of $g$-modules relative to the pair $(g, p)$ (see, e.g., [1]). The following result is due to Shelton, and we refer the reader to [17] for its proof. He defines for any $u$ and $v$ in 
$W^{J}$, a polynomial $E_{u, v}(q)$ by

$$
E_{u, v}(q)=\sum_{k \geq 0}(-1)^{l(v)-l(u)-k} q^{k} \operatorname{dim}\left(\operatorname{Ext}^{k}\left(N_{u}, N_{v}\right)\right)
$$

and proves that

Theorem 2.1. Suppose that $(g, p)$ corresponds to an indecomposable Hermitian symmetric pair. Then for all $u, v \in W^{J}$ :

i) $E_{u, v}(q)=0$ if $u \not \leq v$;

ii) $E_{u, u}(q)=1$;

iii) if $u<v$ and $s \notin D(u)$ with $u s \in W^{J}$, then

$$
E_{u, v}(q)= \begin{cases}E_{u s, v s}(q) & \text { if } s \notin D(v) \text { and } v s \in W^{J} ; \\ (q-1) E_{u s, v}(q) & \text { if } s \in D(v) \text { and us } \leq v s ; \\ \left(q-q^{-1}\right) E_{u s, v}(q)+E_{u s, v s}(q) & \text { if } u \leq u s \leq v s \leq v ; \\ q E_{u s, v}(q) & \text { if } v s \notin W^{J} .\end{cases}
$$

In this paper we solve these recurrence relations in the cases when $(g, p)$ is an indecomposable classic Hermitian symmetric pair (see Table 1). Our main results are explicit product formulas for these polynomials. Moreover, these formulas imply that the polynomials are combinatorial invariants.

TABle 1. The indecomposable classic Hermitian Symmetric pairs $(g, p)$.

$$
\begin{array}{ccc}
(g, p) & g & {[m, m]} \\
S U(r, s) & A_{N} & A_{r-1} \times A_{s-1} \\
S O(2 n-1,2) & B_{n} & B_{n-1} \\
S p(2 n, \mathbf{R}) & C_{n} & A_{n-1} \\
S O(2 n-2,2) & D_{n} & D_{n-1} \\
S O^{*}(2 n) & D_{n} & A_{n-1}
\end{array}
$$

\section{The CASE $\left(A_{n-1}, A_{i-1} \times A_{n-i-1}\right)$}

In this section, we give an explicit product formula for the polynomials $E_{u, v}(q)$ in the case of the pair $\left(A_{n-1}, A_{i-1} \times A_{n-i-1}\right)$. We give two different formulations of this result, one in terms of permutations and one in terms of partitions. Throughout this section we fix $n \in \mathbf{P}$ and $i \in[n-1]$, and we let $W:=S_{n}, s_{i}:=(i, i+1)$ for $i=1, \ldots, n-1, S:=\left\{s_{1}, \ldots, s_{n-1}\right\}$, and $J:=S \backslash\left\{s_{i}\right\}$. It is well known that $\left(S_{n}, S\right)$ is a Coxeter system of type $A_{n-1}$ and that the following characterization holds (see, e.g., [15]).

Proposition 3.1. Let $v \in S_{n}$. Then

$$
D(v)=\left\{s_{i} \in S: v(i)>v(i+1)\right\} .
$$

For symmetric groups, the parabolic subgroups of $S_{n}$ are called Young subgroups. In the case of maximal parabolic subgroups (i.e. $J=S \backslash\left\{s_{i}\right\}$ ) their quotients take this particularly simple form:

$$
W^{J}=\left\{w \in W: w^{-1}(1)<\ldots<w^{-1}(i) \text { and } w^{-1}(i+1)<\ldots<w^{-1}(n)\right\} .
$$

Given $v \in W^{J}$ we associate to $v$ the partition

$$
\Lambda(v):=\left(v^{-1}(i)-i, \ldots, v^{-1}(2)-2, v^{-1}(1)-1\right) .
$$




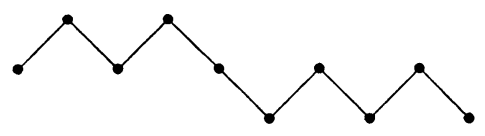

Figure 2. The lattice path corresponding to $(5,4,2,1)$ if $n=9$ and $i=4$.

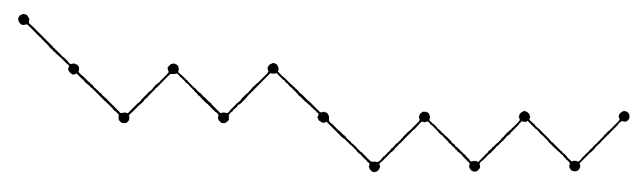

Figure 3 . The lattice path corresponding to $(5,4,2,1)$ if $n=12$ and $i=5$.

The following is well known (see [4 Proposition 2.8]).

Proposition 3.2. The map $\Lambda$ defined by (3) is a bijection between $W^{J}$ and $\mathcal{P}(n, i)$. Furthermore $u \leq v$ in $W^{J}$ if and only if $\Lambda(u) \subseteq \Lambda(v)$, and $l(v)=|\Lambda(v)|$ for all $u, v \in W^{J}$.

We will find it sometimes convenient to identify a partition $\lambda \in \mathcal{P}(n, i)$ with a lattice path, with $(1,1)$ and $(1,-1)$ steps. This path is the union of the lower boundary of the diagram of the skew-partition $(n-i)^{i} \backslash \lambda$ and the upper boundary of the partition $\lambda$. Note that it starts at $(0,0)$ and ends at $(n, 2 i-n)$ (equivalently, it has $n$ steps and exactly $i$ are $(1,1)$-steps). We call a $(1,1)$-step (respectively, $(1,-1)$-step) an up-step (respectively, down-step). Given $j \in[n-1]$ we say that $\lambda$ has a peak at $j$ if the $j$-th step of $\lambda$ is up and its $(j+1)$-th step is down. For example, if we take the partition of Figure $1, \lambda=(5,4,2,1) \in \mathcal{P}(9,4)$, then the associated path is the one shown in Figure 2 and it has peaks at 1,3,6, and 8. Note that this identification between partitions and paths depends on $n$ and $i$. For example, the same partition $(5,4,2,1)$ corresponds to the path in Figure 3 if $n=12$ and $i=5$. Since $n$ and $i$ are fixed throughout this section, this will cause no confusion.

The following elementary lemma is known (see [4. Lemma 2.9]).

Lemma 3.3. Let $v \in W^{J}$, and $j \in[n-1]$. Then $s_{j} \in D(v)$ if and only if $\Lambda(v)$ has a peak at $n-j$.

Note that the $k$-th step of $\Lambda(v)$ is an up step if and only if

$$
k \in\left\{n+1-v^{-1}(i), n+1-v^{-1}(i-1), \ldots, n+1-v^{-1}(1)\right\} .
$$

Let $u, v \in W^{J}, u \leq v$. For $j \in[n]$ we let, following [4],

$$
a_{j}(u, v):=\left|\left\{r \in u^{-1}([i]): r<j\right\}\right|-\left|\left\{r \in v^{-1}([i]): r<j\right\}\right| .
$$

For example, if $n=9, i=4, u=123564789$, and $v=516278394$, then

$$
\left(a_{1}(u, v), \ldots, a_{9}(u, v)\right)=(0,1,1,2,1,1,2,1,1) .
$$

Note that it follows easily from Proposition 3.2 that $a_{j}(u, v) \geq 0$ for $j=1, \ldots, n$ if and only if $u \leq v$, and that $a_{j}(u, v)>0$ if $j \in v^{-1}([i]) \backslash u^{-1}([i])$ and $u \leq v$. Also note that, if $u \in W^{J}$ and $j \in[n]$, then

$$
\left|\left\{r \in u^{-1}([i]): r<j\right\}\right|= \begin{cases}u(j)-1, & \text { if } j \in u^{-1}([i]), \\ j+i-u(j), & \text { if } j \notin u^{-1}([i]) .\end{cases}
$$


This may be used to obtain a more explicit formula for $a_{j}(u, v)$, if desired.

We can now state and prove the main result of this section.

Theorem 3.4. Let $u, v \in W^{J}, u \leq v$. Then

$$
E_{u, v}(q)=q^{l(v)-l(u)} \prod_{j \in v^{-1}([i]) \backslash u^{-1}([i])}\left(1-q^{-2 a_{j}(u, v)+1}\right) .
$$

Proof. Let, for brevity, $D_{i}(u, v):=v^{-1}([i]) \backslash u^{-1}([i])$. We proceed by induction on $l\left(w_{0}^{J}\right)-l(u)$. If $l\left(w_{0}^{J}\right)-l(u)=0$, we have $w_{0}^{J}=v=u$ and the result is trivially true. So suppose that $l\left(w_{0}^{J}\right)-l(u)>0$ and let $s=(k, k+1)$ be such that $s \notin D(u)$ and $u s \in W^{J}$. Note that, since $u \in W^{J}$, this implies that $k \in u^{-1}([i])$ and $k+1 \notin u^{-1}([i])$. We have four cases to consider.

a) $s \notin D(v)$, and $v s \in W^{J}$.

Since $v \in W^{J}$, this implies that $k \in v^{-1}([i])$ and $k+1 \notin v^{-1}([i])$. Moreover, $u s, v s \in W^{J}$ and so $D_{i}(u s, v s)=D_{i}(u, v)$ and $a_{j}(u s, v s)=a_{j}(u, v)$ for all $j \in[n]$, since $(u s)^{-1}([i])$ is obtained from $u^{-1}([i])$ by replacing $k$ by $k+1$, and similarly for $v$. Hence, by Theorem 2.1 and our induction hypothesis,

$$
\begin{aligned}
E_{u, v}(q) & =E_{u s, v s} \\
& =q^{l(v s)-l(u s)} \prod_{j \in D_{i}(u s, v s)}\left(1-q^{-2 a_{j}(u s, v s)+1}\right) \\
& =q^{l(v)-l(u)} \prod_{j \in D_{i}(u, v)}\left(1-q^{-2 a_{j}(u, v)+1}\right),
\end{aligned}
$$

as desired.

b) $s \in D(v)$, and $u s \not \leq v s$.

Since $v \in W^{J}$ this implies that $k \notin v^{-1}([i]), k+1 \in v^{-1}([i])$, and $v s \in W^{J}$. So we have that $D_{i}(u s, v)=D_{i}(u, v) \backslash\{k+1\}$,

$$
a_{j}(u s, v)=a_{j}(u, v)=a_{j}(u s, v s)
$$

for all $j \in[n] \backslash\{k+1\}$, and

$$
a_{k+1}(u s, v s)=a_{k+1}(u, v)-2 .
$$

Since $u s \not \leq v s$ by (8) and the note before the statement of this theorem, we conclude that $a_{k+1}(u, v)=1$. Hence, by Theorem 2.1 and the induction hypothesis,

$$
\begin{aligned}
E_{u, v}(q) & =(q-1) E_{u s, v}(q) \\
& =(q-1) q^{l(v)-l(u)-1} \prod_{j \in D_{i}(u, v) \backslash\{k+1\}}\left(1-q^{-2 a_{j}(u s, v)+1}\right) \\
& =\frac{(q-1) q^{l(v)-l(u)-1}}{\left(1-q^{-2 a_{k+1}(u, v)+1}\right)} \prod_{j \in D_{i}(u, v)}\left(1-q^{-2 a_{j}(u, v)+1}\right) \\
& =q^{l(v)-l(u)} \prod_{j \in D_{i}(u, v)}\left(1-q^{-2 a_{j}(u, v)+1}\right),
\end{aligned}
$$

as desired. 
c) $s \in D(v)$, and $u s \leq v s$.

Since $v \in W^{J}$ this implies that $k \notin v^{-1}([i]), k+1 \in v^{-1}([i])$, and $v s \in W^{J}$. So we have that

$$
\begin{gathered}
D_{i}(u s, v)=D_{i}(u, v) \backslash\{k+1\}, \\
D_{i}(u s, v s)=\left(D_{i}(u, v) \backslash\{k+1\}\right) \cup\{k\},
\end{gathered}
$$

$a_{j}(u s, v)=a_{j}(u s, v s)=a_{j}(u, v)$ for all $j \in[n] \backslash\{k+1\}, a_{k+1}(u s, v s)=a_{k+1}(u, v)-2$ and $a_{k+1}(u, v)=a_{k}(u, v)+1$. Hence, by Theorem 2.1 and our induction hypothesis,

$$
\begin{aligned}
E_{u, v}(q)= & \left(q-q^{-1}\right) E_{u s, v}(q)+E_{u s, v s}(q) \\
= & \left(q-q^{-1}\right) q^{l(v)-l(u)-1} \prod_{j \in D_{i}(u, v) \backslash\{k+1\}}\left(1-q^{-2 a_{j}(u s, v)+1}\right) \\
& +q^{l(v)-l(u)-2} \prod_{j \in\left(D_{i}(u, v) \backslash\{k+1\}\right) \cup\{k\}}\left(1-q^{-2 a_{j}(u s, v s)+1}\right) \\
= & \left(q-q^{-1}\right) q^{l(v)-l(u)-1} \prod_{j \in D_{i}(u, v) \backslash\{k+1\}}\left(1-q^{-2 a_{j}(u, v)+1}\right) \\
& +q^{l(v)-l(u)-2}\left(1-q^{-2 a_{k}(u, v)+1}\right) \\
= & q^{l(v)-l(u)-2} \frac{\left(q^{2}-q^{-2 a_{k}(u, v)+1}\right)}{\left(1-q^{-2 a_{k+1}(u, v)+1}\right)} \prod_{j \in D_{i}(u, v) \backslash\{k+1\}}\left(1-q^{-2 a_{j}(u, v)+1}\right) \\
= & q^{l(v)-l(u)} \prod_{j \in D_{i}(u, v)}\left(1-q^{-2 a_{j}(u, v)+1}\right)
\end{aligned}
$$

and the result again follows.

d) $v s \notin W^{J}$.

Then $s \notin D(v)$ and we have two cases. In the first one, we have $k, k+1 \in v^{-1}([i])$, which implies that $D_{i}(u s, v)=\left(D_{i}(u, v) \backslash\{k+1\}\right) \cup\{k\}$ and $a_{j}(u s, v)=a_{j}(u, v)$ for $j \in[n] \backslash\{k+1\}, a_{k+1}(u, v)=a_{k}(u s, v)$. Hence, by Theorem 2.1 and the induction hypothesis,

$$
\begin{aligned}
E_{u, v}(q) & =q E_{u s, v}(q) \\
& =q^{l(v)-l(u)} \prod_{j \in\left(D_{i}(u, v) \backslash\{k+1\}\right) \cup\{k\}}\left(1-q^{-2 a_{j}(u s, v)+1}\right) \\
& =q^{l(v)-l(u)} \frac{\left(1-q^{-2 a_{k}(u s, v)+1}\right)}{\left(1-q^{-2 a_{k+1}(u, v)+1}\right)} \prod_{j \in D_{i}(u, v)}\left(1-q^{-2 a_{j}(u, v)+1}\right) \\
& =q^{l(v)-l(u)} \prod_{j \in D_{i}(u, v)}\left(1-q^{-2 a_{j}(u, v)+1}\right) .
\end{aligned}
$$

In the second case we have, $k, k+1 \notin v^{-1}([i])$, hence $D_{i}(u s, v)=D_{i}(u, v)$ and $a_{j}(u s, v)=a_{j}(u, v)$ for $j \in[n] \backslash\{k+1\}$. Hence, by Theorem[2.1 and the induction 
hypothesis,

$$
\begin{aligned}
E_{u, v}(q) & =q E_{u s, v}(q) \\
& =q^{l(v)-l(u)} \prod_{j \in D_{i}(u, v)}\left(1-q^{-2 a_{j}(u s, v)+1}\right) \\
& =q^{l(v)-l(u)} \prod_{j \in D_{i}(u, v)}\left(1-q^{-2 a_{j}(u, v)+1}\right),
\end{aligned}
$$

and the result again follows.

This completes the induction step and hence the proof.

Because $W^{J}$ is isomorphic, as a poset, to a lower interval in Young's lattice (by Proposition 3.2), it is natural to rephrase Theorem 3.4 in the language of partitions rather than permutations.

Let $\mu, \lambda \in \mathcal{P}(n, i)$, with $\mu \subseteq \lambda$. Think of $\mu$ and $\lambda$ as paths as explained above. Then, by Proposition 3.2, the path $\lambda$ lies (weakly) above the path $\mu$. Let $1 \leq j \leq n$ and consider the $j$-th step of $\lambda$ (from the left). Following [4, we say that such a step is allowable with respect to $\mu$ if it is an up-step and the $j$-th step of $\mu$ is a down-step. For example, if $n=9, i=4, \lambda=(5,4,2,1)$, and $\mu=(2,0,0,0)$, then the $j$-th step of $\lambda$ is allowable with respect to $\mu$ exactly if $j \in\{1,3,6\}$ (see Figure 4). Now let $\tilde{a}_{j}(\mu, \lambda)$ be the vertical distance (divided by two, since it is always even) between the (right end of the) $j$-th step of $\lambda$ and the (right end of the) $j$-th step of $\mu$. We then have the following result, and we refer the reader to 4, Proposition 3.3] for its proof.

Proposition 3.5. Let $u, v \in W^{J}, u \leq v$. Then

$$
a_{j}(u, v)=\tilde{a}_{n+1-j}(\Lambda(u), \Lambda(v))
$$

for $j=1, \ldots, n$. Furthermore $n+1-j \in v^{-1}([i]) \backslash u^{-1}([i])$ if and only if the $j$-th step of $\Lambda(v)$ is allowable with respect to $\Lambda(u)$.

We can now rephrase Theorem 3.4 in terms of partitions.

Corollary 3.6. Let $u, v \in W^{J}, u \leq v$. Then

$$
E_{u, v}(q)=q^{|\lambda \backslash \mu|} \prod_{j}\left(1-q^{-2 \tilde{a}_{j}(\mu, \lambda)+1}\right),
$$

where $\mu=\Lambda(u), \lambda=\Lambda(v)$ and $j$ runs over all the allowable steps of $\lambda$ with respect to $\mu$. In particular, $E_{u, v}(q)$ depends only on $\Lambda(v) \backslash \Lambda(u)$.

In the case of a lower interval, formula (9) takes up a particularly simple form. The proof of the next result is analogous to the one of [4, Corollary 3.5], and we leave it to the reader.

Corollary 3.7. Let $v \in W^{J}$. Then

$$
E_{e, v}(q)=q^{|\mu|} \prod_{j=1}^{d(\mu)}\left(1-q^{-2 j+1}\right),
$$

where $\mu=\Lambda(v)$ and $d(\mu)$ is the length of the Durfee square of $\mu$. 


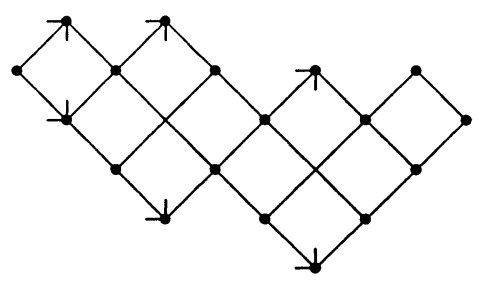

FIGURE 4.

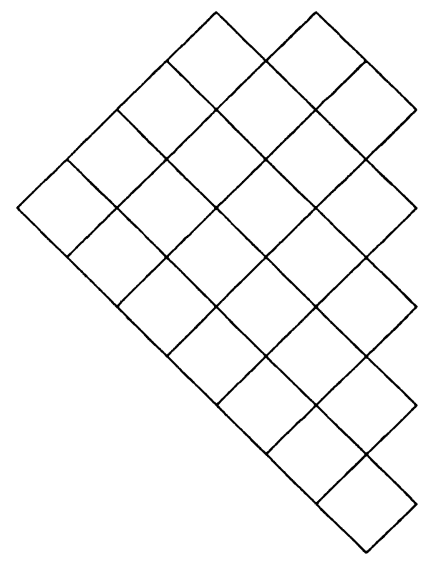

FIGURE 5.

We close this section with an example to illustrate Theorem 3.4 and Corollary 3.6. We use the various calculations made in $\S 2$ and $\S 3$. Let $u=123564789$ and $v=516278394$ in $S_{9}^{S \backslash\{(4,5)\}}$; we have $D_{4}(u, v)=\{4,7,9\}$. From (5) and (6), it follows that

$$
E_{u, v}(q)=q^{10}\left(1-q^{-1}\right)\left(1-q^{-3}\right)\left(1-q^{-3}\right) .
$$

Observe that we have $\Lambda(v)=(5,4,2,1)=\lambda$ and $\Lambda(u)=(2,0,0,0)=\mu$. The diagram of the skew-partition $\Lambda(v) \backslash \Lambda(u)$ is drawn in Figure 4 . The allowable are indicated by arrows and $\tilde{a}_{1}(\mu, \lambda)=1, \tilde{a}_{3}(\mu, \lambda)=2, \tilde{a}_{6}(\mu, \lambda)=2$.

$$
\text { 4. The CASE }\left(C_{n}, A_{n-1}\right)
$$

A partition $\left(\lambda_{1}, \lambda_{2}, \ldots, \lambda_{k}\right)$ is strict if $\lambda_{1}>\lambda_{2}>\ldots>\lambda_{k}$. We denote by $\mathcal{P}_{s}$ the set of all (integer) strict partitions. Let

$$
H:=\left\{(i, j) \in \mathbf{P}^{2}: i \leq j\right\}
$$

with the ordering induced by the product ordering on $\mathbf{P}^{2}$. We call the finite order ideals of $H$ shifted partitions. Denote by $\mathcal{I}$ the set of all finite order ideals of $H$. Note that $\mathcal{I}$ is partially ordered by set inclusion. It is well known that this makes $\mathcal{I}$ into a distributive lattice. We identify a shifted partition with its diagram

$$
\left\{(i, j) \in \mathbf{P}^{2}: 1 \leq i \leq k, i \leq j \leq \lambda_{i}-1+i\right\},
$$

and as in $\S 3$ we draw it rotated counterclockwise by $\frac{\pi}{4}$ radians. So for example the diagram of $(7,6,5,4,2)$ is illustrated in Figure 5 . 
Let

$$
\tilde{\mathcal{P}}:=\left\{\lambda \in \mathcal{P}: \lambda \supseteq l(\lambda)^{l(\lambda)}\right\} .
$$

Note that there are inclusion preserving bijections between strict partitions, shifted partitions and partitions in $\tilde{\mathcal{P}}$, given by

$$
\begin{aligned}
\left(\lambda_{1}, \lambda_{2}, \ldots, \lambda_{k}\right) & \leftrightarrow\left\{(i, j) \in \mathbf{P}^{2}: 1 \leq i \leq k, i \leq j \leq \lambda_{i}-1+i\right\} \\
& \leftrightarrow\left(\lambda_{1}, \lambda_{2}+1, \ldots, \lambda_{k}+k-1\right) .
\end{aligned}
$$

For this reason, we will freely identify these objects. Note, however, that only the bijection between strict partitions and shifted partitions preserves size. In fact, if $\lambda, \mu, \in \tilde{\mathcal{P}}$ and $\nu \in \mathcal{P}$, with $\mu \subseteq \nu \subseteq \lambda$, then it does not necessarily follow that $\nu \in \tilde{\mathcal{P}}$. Therefore the subposet

$$
\{\nu \in \mathcal{P}: \mu \subseteq \nu \subseteq \lambda\}
$$

of $\mathcal{P}$ is not isomorphic to the subposet

$$
\{\nu \in \mathcal{I}: \mu \subseteq \nu \subseteq \lambda\}
$$

of $\mathcal{I}$.

Our purpose in this section is to obtain an explicit product formula for the $E$ polynomials in the case of the pair $\left(C_{n}, A_{n-1}\right)$. Therefore, let $S_{n}^{B}$ be the group of all the bijections of the set $[ \pm n]$ in itself such that

$$
w(-a)=-w(a)
$$

for all $a \in[ \pm n]$, with composition as group operation. If $w \in S_{n}^{B}$, then, following [6], we write $w=\left[a_{1}, \ldots, a_{n}\right]$ to mean that $w(i)=a_{i}$, for $i=1, \ldots, n$, and call this the window notation of $w$. Because of this notation the group $S_{n}^{B}$ is often called the group of all signed permutations on $[n]$. Since the elements of $S_{n}^{B}$ are permutations of $[ \pm n]$ we can also write them in disjoint cycle form; as in $\S 2$, we multiply elements "from the right". We identify $S_{n}$ as a subgroup of $S_{n}^{B}$ in the natural way. For the rest of this section, we fix $n \in \mathbf{P}$ and we let $W:=S_{n}^{B}, s_{i}:=(i, i+1)(-i-1,-i)$ for $i=1, \ldots, n-1, s_{0}:=(1,-1), S:=\left\{s_{0}, s_{1}, \ldots, s_{n-1}\right\}$ and $J:=S \backslash\left\{s_{0}\right\}$. It is well known that $\left(S_{n}^{B}, S\right)$ is a Coxeter system of type $B_{n}$ and that the following characterization holds (see, e.g., [6]).

Proposition 4.1. Let $v \in S_{n}^{B}$. Then

$$
D(v)=\left\{s_{i} \in S: v(i)>v(i+1)\right\},
$$

where $v(0):=0$.

From Proposition 4.1 we have that

$$
W^{J}=\left\{v \in W: v^{-1}(1)<v^{-1}(2)<\ldots<v^{-1}(n)\right\} .
$$

Therefore, given $v \neq e, v \in W^{J}$, there is a unique $k \in[n]$ (in fact, $k=N_{1}(v)$ ) such that

$$
v^{-1}(k)<0<v^{-1}(k+1)
$$

and we associate to $v$ the shifted partition

$$
\Lambda_{B}(v):=\left(-v^{-1}(1),-v^{-1}(2), \ldots,-v^{-1}(k)\right) .
$$

Let

$$
\tilde{\mathcal{I}}(n):=\{\lambda \in \mathcal{I}: \lambda \subseteq(n, n-1, \ldots, 2,1)\} .
$$




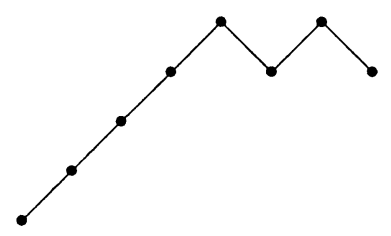

FiguRE 6.

The following is known:

Proposition 4.2. The map $\Lambda_{B}$ defined by (12) is a bijection between $W^{J}$ and $\tilde{\mathcal{I}}(n)$. Furthermore $u \leq v$ in $W^{J}$ if and only if $\Lambda_{B}(u) \subseteq \Lambda_{B}(v)$ and $l(v)=\left|\Lambda_{B}(v)\right|$ for all $u, v \in W^{J}$.

As before it is convenient to identify a shifted partition $\lambda \in \tilde{\mathcal{I}}(n)$ with a lattice path with $(1,1)$ and $(1,-1)$ steps starting at $(0,0)$ and having $n$ steps. We have the obvious bijection between the peaks of $\lambda$ as a path and the upper peaks of $\lambda$ as a partition. Note that, as in Proposition 3.2 this bijection depends on $n$, but for us $n$ is fixed and so there is no confusion. For example, the partition $\lambda=(7,6,5,4,2)$ corresponds to the path in Figure 6.

Lemma 4.3. Let $v \in W^{J}$ and $j \in[n-1]$. Then $s_{j} \in D(v)$ if and only if $\Lambda_{B}(v)$ has a peak at $n-j$. Furthermore, $s_{o} \in D(v)$ if and only if the last step of $\Lambda_{B}(v)$ is up.

This result can be proved in a way similar to Lemma 3.3 (see also Lemma 5.3) and is due to Brenti [5]. Note that $i$-th step of $\Lambda_{B}(v)$ is an up-step if and only if

$$
i \in\left\{n+1+v^{-1}(1), n+1+v^{-1}(2), \ldots, n+1+v^{-1}(k)\right\} .
$$

Proposition 4.4. Let $v \in W^{J}$ and $i \in[n]$. Then the $i$-th step (from the left) of $\Lambda_{B}(v)$ (seen as a path) is an up-step if and only if $v(n+1-i)<0$.

Proof. We know that the $i$-th step of $v \in W^{J}$ is an up-step if and only if

$$
n+1-i \in\left\{-v^{-1}(1),-v^{-1}(2), \ldots,-v^{-1}(k)\right\} .
$$

But this, by the definition of $k$, happens if and only if $v(n+1-i)<0$, as desired.

We are now ready to prove the main theorem of this section, which gives an explicit product formula for the polynomials $E_{u, v}(q)$ in the case of the symmetric pair $\left(C_{n}, A_{n-1}\right)$. As in the preceding section we give two different formulations of this result, one in terms of signed permutations and one in terms of shifted partitions.

Let $u, v \in W^{J}, u \leq v$. For $j \in[n]$ let, following [5],

$$
b_{j}(u, v):=|\{r \geq j: v(r)<0\}|-|\{r \geq j: u(r)<0\}| .
$$

For example, if $u=[4,5,-3,-2,6,7,-1]$ and $v=[6,-5,7,-4,-3,-2,-1]$, then

$$
\left(b_{1}(u, v), \ldots, b_{7}(u, v)\right)=(2,2,1,2,2,1,0) .
$$

Note that it follows from Proposition 4.2 that $b_{j}(u, v) \geq 0$ for $j=1, \ldots, n$ if and only if $u \leq v$. Also, if $u \leq v$, then $b_{j}(u, v)>0$ when $v(j)<0<u(j)$. We let

$$
N(u, v):=\{r \in[n]: u(r) v(r)<0\}
$$


and

$$
D(u, v):=\left\{r \in N(u, v):(-1)^{b_{r}(u, v)}<0\right\} .
$$

Theorem 4.5. Let $u, v \in W^{J}, u \leq v$. Then

$$
E_{u, v}(q)=q^{l(v)-l(u)} \prod_{j \in D(u, v)}\left(1-q^{-\tilde{b}_{j}(u, v)}\right)
$$

where

$$
\tilde{b}_{j}(u, v):= \begin{cases}2 b_{j}(u, v)-1 & \text { if } u(j)>0 \\ 2 b_{j}(u, v)+1 & \text { if } u(j)<0\end{cases}
$$

Proof. We proceed by induction on $l\left(w_{0}^{J}\right)-l(u)$. If $l\left(w_{0}^{J}\right)-l(u)=0$, we have $w_{0}^{J}=v=u$ and the result is trivially true. So suppose that $l\left(w_{0}^{J}\right)-l(u)>0$ and let $s$ be such that $s \notin D(u)$ and $u s \in W^{J}$.

Suppose first that $s=(-i-1,-i)(i, i+1)$ for some $i \in[n-1]$; then $u(i)<u(i+1)$. Note that, since $u \in W^{J}$, this implies that $u(i)<0<u(i+1)$. We have four cases to consider.

a) $s \notin D(v)$, and $v s \in W^{J}$.

Since $v \in W^{J}$, this implies that $v(i)<0<v(i+1)$. Moreover $u s, v s \in W^{J}$, and so $N(u s, v s)=N(u, v)$ and $b_{j}(u s, v s)=b_{j}(u, v)$ for all $j \in[n]$, so that $D(u s, v s)=$ $D(u, v)$ and $\tilde{b}_{j}(u s, v s)=\tilde{b}_{j}(u, v)$ for all $j \in[n] \backslash\{i, i+1\}$. Hence, by Theorem 2.1 and our induction hypothesis,

$$
\begin{aligned}
E_{u, v}(q) & =E_{u s, v s}(q) \\
& =q^{l(v s)-l(u s)} \prod_{j \in D(u s, v s)}\left(1-q^{-\tilde{b}_{j}(u s, v s)}\right) \\
& =q^{l(v)-l(u)} \prod_{j \in D(u, v)}\left(1-q^{-\tilde{b}_{j}(u, v)}\right),
\end{aligned}
$$

as desired.

b) $s \in D(v)$, and $u s \not \leq v s$.

Then $v(i)>0>v(i+1)$, therefore $N(u s, v)=N(u, v) \backslash\{i, i+1\}, b_{j}(u s, v)=$ $b_{j}(u, v)=b_{j}(u s, v s)$ for all $j \in[n] \backslash\{i+1\}$, and

$$
b_{i}(u, v)=b_{i+1}(u, v)-1
$$

Since $u s \not \leq v s$, it follows from the note before the statement of the theorem that $b_{i+1}(u, v)>0>b_{i+1}(u s, v s)$. Also $b_{i+1}(u, v)-b_{i+1}(u s, v s)=2$, so $b_{i+1}(u, v)=$ 1. This and (21) imply that $i \notin D(u, v)$ and $i+1 \in D(u, v)$. It follows that $D(u s, v)=D(u, v) \backslash\{i+1\}, \tilde{b}_{j}(u s, v)=\tilde{b}_{j}(u, v)$ for all $j \in[n] \backslash\{i, i+1\}$, and that $\tilde{b}_{i+1}(u, v)=2 b_{i+1}(u, v)-1=1$. Hence, by Theorem 2.1 and the induction 
hypothesis,

$$
\begin{aligned}
E_{u, v}(q) & =(q-1) E_{u s, v}(q) \\
& =(q-1) q^{l(v)-l(u)-1} \prod_{j \in D(u, v) \backslash\{i+1\}}\left(1-q^{-\tilde{b}_{j}(u s, v)}\right) \\
& =\frac{(q-1) q^{l(v)-l(u)-1}}{\left(1-q^{-\tilde{b}_{i+1}(u, v)}\right)} \prod_{j \in D(u, v)}\left(1-q^{-\tilde{b}_{j}(u, v)}\right) \\
& =q^{l(v)-l(u)} \prod_{j \in D(u, v)}\left(1-q^{-\tilde{b}_{j}(u, v)}\right) .
\end{aligned}
$$

c) $s \in D(v)$, and $u s \leq v s$.

Then, as above, $v(i)>0>v(i+1), N(u s, v)=N(u, v) \backslash\{i, i+1\}, b_{j}(u s, v)=$ $b_{j}(u, v)$ for all $j \in[n] \backslash\{i+1\}$, and

$$
b_{i}(u, v)=b_{i+1}(u, v)-1 .
$$

It follows that $D(u s, v)=D(u, v) \backslash\{i, i+1\}$ and $\tilde{b}_{j}(u s, v)=\tilde{b}_{j}(u, v)$, for all $j \in$ $[n] \backslash\{i, i+1\}$. On the other hand, we have $N(u s, v s)=N(u, v)$ and $b_{j}(u s, v s)=$ $b_{j}(u, v)$ for all $j \in[n] \backslash\{i+1\}$, and $b_{i+1}(u s, v s)=b_{i+1}(u, v)-2$. It follows that $D(u s, v s)=D(u, v)$ and $\tilde{b}_{j}(u s, v s)=\tilde{b}_{j}(u, v)$ for all $j \in[n] \backslash\{i, i+1\}$. Hence, by Theorem 2.1 and the induction hypothesis,

$$
\begin{aligned}
E_{u, v}(q)= & \left(q-q^{-1}\right) E_{u s, v}(q)+E_{u s, v s}(q) \\
= & \left(q-q^{-1}\right) q^{l(v)-l(u)-1} \prod_{j \in D(u, v) \backslash\{i, i+1\}}\left(1-q^{-\tilde{b}_{j}(u s, v)}\right) \\
& +q^{l(v)-l(u)-2} \prod_{j \in D(u, v)}\left(1-q^{-\tilde{b}_{j}(u s, v s)}\right) .
\end{aligned}
$$

From (22) we have that $i \in D(u, v)$ if and only if $i+1 \notin D(u, v)$, so we have two cases. If $i \in D(u, v)$, then $D(u s, v)=D(u, v) \backslash\{i\}$ and $\tilde{b}_{i}(u s, v s)=2 b_{i}(u s, v s)-1=$ $2 b_{i}(u, v)-1=\tilde{b}_{i}(u, v)-2$ because $u(i)<0<u(i+1)$. Hence, by (23)),

$$
\begin{aligned}
E_{u, v}(q)= & \left(q^{2}-1\right) q^{l(v)-l(u)-2} \prod_{j \in D(u, v) \backslash\{i\}}\left(1-q^{-\tilde{b}_{j}(u, v)}\right) \\
& +q^{l(v)-l(u)-2}\left(1-q^{-\tilde{b}_{i}(u s, v s)}\right) \prod_{j \in D(u, v) \backslash\{i\}}\left(1-q^{-\tilde{b}_{j}(u, v)}\right) \\
= & q^{l(v)-l(u)-2} \frac{\left(q^{2}-q^{-\tilde{b}_{i}(u s, v s)}\right)}{\left(1-q^{-\tilde{b}_{i}(u, v)}\right)} \prod_{j \in D(u, v)}\left(1-q^{-\tilde{b}_{j}(u, v)}\right) \\
= & q^{l(v)-l(u)} \prod_{j \in D(u, v)}\left(1-q^{-\tilde{b}_{j}(u, v)}\right) .
\end{aligned}
$$


If $i+1 \in D(u, v)$, then $D(u s, v)=D(u, v) \backslash\{i+1\}$ and $\tilde{b}_{i+1}(u s, v s)=2 b_{i+1}(u s, v s)$ $+1=\left(2 b_{i+1}(u, v)-1\right)-2=\tilde{b}_{i+1}(u, v)-2$. Hence, by (23),

$$
\begin{aligned}
E_{u, v}(q)= & \left(q^{2}-1\right) q^{l(v)-l(u)-2} \prod_{j \in D(u, v) \backslash\{i+1\}}\left(1-q^{-\tilde{b}_{j}(u, v)}\right) \\
& +q^{l(v)-l(u)-2}\left(1-q^{-\tilde{b}_{i+1}(u s, v s)}\right) \prod_{j \in D(u, v) \backslash\{i+1\}}\left(1-q^{-\tilde{b}_{j}(u, v)}\right) \\
= & q^{l(v)-l(u)-2} \frac{\left(q^{2}-q^{-\tilde{b}_{i+1}(u s, v s)}\right)}{\left(1-q^{-\tilde{b}_{i+1}(u, v)}\right)} \prod_{j \in D(u, v)}\left(1-q^{-\tilde{b}_{j}(u, v)}\right) \\
= & q^{l(v)-l(u)} \prod_{j \in D(u, v)}\left(1-q^{-\tilde{b}_{j}(u, v)}\right),
\end{aligned}
$$

and the result follows also in this case.

d) $v s \notin W^{J}$.

Then $s \notin D(v)$ and we have two cases. In the first one, we have $v(i)<v(i+1)<0$ and this implies that $N(u s, v)=(N(u, v) \backslash\{i+1\}) \cup\{i\}, b_{j}(u s, v)=b_{j}(u, v)$ for all $j \in[n] \backslash\{i+1\}, b_{i}(u s, v)=b_{i+1}(u, v)$. It follows that

$$
D(u s, v) \backslash\{i\}=D(u, v) \backslash\{i+1\},
$$

$i \in D(u s, v)$, if and only if $i+1 \in D(u, v), \tilde{b}_{j}(u s, v)=\tilde{b}_{j}(u, v)$ for all $j \in[n] \backslash\{i, i+1\}$ and $\tilde{b}_{i}(u s, v)=\tilde{b}_{i+1}(u, v)$. Hence, by Theorem 2.1 and the induction hypothesis, if $i \notin D(u s, v), D(u s, v)=D(u, v)$ and the thesis easily follows. Otherwise if $i \in D(u s, v)$, we have

$$
\begin{aligned}
E_{u, v}(q) & =q E_{u s, v}(q) \\
& =q q^{l(v)-l(u)-1} \prod_{j \in D(u s, v)}\left(1-q^{-\tilde{b}_{j}(u s, v)}\right) \\
& =q^{l(v)-l(u)} \frac{\left(1-q^{-\tilde{b}_{i}(u s, v)}\right)}{\left(1-q^{-\tilde{b}_{i+1}(u, v)}\right)} \prod_{j \in D(u, v)}\left(1-q^{-\tilde{b}_{j}(u, v)}\right) \\
& =q^{l(v)-l(u)} \prod_{j \in D(u, v)}\left(1-q^{-\tilde{b}_{j}(u, v)}\right),
\end{aligned}
$$

because $\tilde{b}_{i}(u s, v)=\tilde{b}_{i+1}(u, v)$.

In the second case, we have $0<v(i)<v(i+1)$ and this implies that $N(u s, v)=$ $(N(u, v) \backslash\{i\}) \cup\{i+1\}, b_{j}(u s, v)=b_{j}(u, v)$ for all $j \in[n] \backslash\{i+1\}, b_{i+1}(u s, v)=$ $b_{i}(u, v)$. It follows that

$$
D(u s, v) \backslash\{i+1\}=D(u, v) \backslash\{i\},
$$

$i+1 \in D(u s, v)$, if and only if $i \in D(u, v), \tilde{b}_{j}(u s, v)=\tilde{b}_{j}(u, v)$ for all $j \in[n] \backslash\{i, i+1\}$ and $\tilde{b}_{i+1}(u s, v)=\tilde{b}_{i}(u, v)$. Hence, by Theorem 2.1 and the induction hypothesis, if $i+1 \notin D(u s, v), D(u s, v)=D(u, v)$ and the thesis easily follows. Otherwise if 
$i+1 \in D(u s, v)$, we have

$$
\begin{aligned}
E_{u, v}(q) & =q E_{u s, v}(q) \\
& =q^{l(v)-l(u)} \prod_{j \in D(u s, v)}\left(1-q^{-\tilde{b}_{j}(u s, v)}\right) \\
& =q^{l(v)-l(u)} \frac{\left(1-q^{-\tilde{b}_{i+1}(u s, v)}\right)}{\left(1-q^{-\tilde{b}_{i}(u, v)}\right)} \prod_{j \in D(u, v)}\left(1-q^{-\tilde{b}_{j}(u, v)}\right),
\end{aligned}
$$

since $\tilde{b}_{i+1}(u s, v)=\tilde{b}_{i}(u, v)$, the result again follows.

Suppose now that $s=(-1,1)=s_{0}$. Then $u(1)>0$, and we observe that $u s, v s \in W^{J}$. We therefore have three cases to consider.

$$
\text { 1) } s \notin D(v) \text {. }
$$

Then $v s \in W^{J}$ and $v(1)>0$. Hence $N(u s, v s)=N(u, v), b_{j}(u s, v s)=b_{j}(u, v)$ for all $j \in[n]$ and so $D(u s, v s)=D(u, v), 1 \notin D(u, v), \tilde{b}_{j}(u s, v s)=\tilde{b}_{j}(u, v)$, for all $j \in[n] \backslash\{1\}$ and the result follows from Theorem 2.1 and the induction hypothesis.

2) $s \in D(v)$, and $u s \not \leq v s$.

Then $v(1)<0$ so $N(u s, v)=N(u, v) \backslash\{1\}, b_{j}(u s, v)=b_{j}(u, v)$ for all $j \in[n] \backslash\{1\}$. Therefore $D(u s, v)=D(u, v) \backslash\{1\}$ and $\tilde{b}_{j}(u s, v)=\tilde{b}_{j}(u, v)$ for all $j \in[n] \backslash\{1\}$. Also, $u<v$ and $u s \not \leq v s$, so by the remark before the statement of the theorem, $b_{1}(u, v)=1$ and hence $\tilde{b}_{1}(u, v)=1$. Hence,

$$
\begin{aligned}
E_{u, v}(q) & =(q-1) E_{u s, v}(q) \\
& =(q-1) q^{l(v)-l(u)-1} \prod_{j \in D(u, v) \backslash\{1\}}\left(1-q^{-\tilde{b}_{j}(u, v)}\right) \\
& =q^{l(v)-l(u)} \prod_{j \in D(u, v)}\left(1-q^{-\tilde{b}_{j}(u, v)}\right) .
\end{aligned}
$$

3) $s \in D(v)$, and $u s \leq v s$.

Then $v(1)<0, N(u s, v s)=N(u, v), N(u s, v)=N(u, v) \backslash\{1\}, b_{j}(u s, v s)=$ $b_{j}(u, v)=b_{j}(u s, v)$ for all $j \in[n] \backslash\{1\}$, and $b_{1}(u s, v s)=b_{1}(u, v)-2=b_{1}(u s, v)-1$. It follows that

$$
D(u s, v)=D(u, v) \backslash\{1\}=D(u s, v s) \backslash\{1\}
$$

and $\tilde{b}_{j}(u s, v s)=\tilde{b}_{j}(u, v)=\tilde{b}_{j}(u s, v)$ for all $j \in[n] \backslash\{1\}$. Hence,

$$
\begin{aligned}
E_{u, v}(q)= & \left(q-q^{-1}\right) E_{u s, v}(q)+E_{u s, v s}(q) \\
= & \left(q-q^{-1}\right) q^{l(v)-l(u)-1} \prod_{j \in D(u, v) \backslash\{1\}}\left(1-q^{-\tilde{b}_{j}(u s, v)}\right) \\
& +q^{l(v)-l(u)-2} \prod_{j \in D(u s, v s)}\left(1-q^{-\tilde{b}_{j}(u s, v s)}\right) .
\end{aligned}
$$


Now we have two cases. If $1 \in D(u, v)$, then $1 \in D(u s, v s)$ and $\tilde{b}_{1}(u s, v s)=$ $\tilde{b}_{1}(u, v)-2$, so from (24),

$$
\begin{aligned}
E_{u, v}(q)= & \left(q^{2}-1\right) q^{l(v)-l(u)-2} \prod_{j \in D(u, v) \backslash\{1\}}\left(1-q^{-\tilde{b}_{j}(u, v)}\right) \\
& +q^{l(v)-l(u)-2}\left(1-q^{-\tilde{b}_{1}(u s, v s)}\right) \prod_{j \in D(u, v) \backslash\{1\}}\left(1-q^{-\tilde{b}_{j}(u, v)}\right) \\
= & q^{l(v)-l(u)} \frac{\left(1-q^{-\tilde{b}_{1}(u s, v s)-2}\right)}{\left(1-q^{-\tilde{b}_{1}(u, v)}\right)} \prod_{j \in D(u, v)}\left(1-q^{-\tilde{b}_{j}(u, v)}\right) .
\end{aligned}
$$

If $1 \notin D(u, v)$, then $1 \notin D(u s, v s)$ and from (24) we have that

$$
\begin{aligned}
E_{u, v}(q)= & \left(q^{2}-1\right) q^{l(v)-l(u)-2} \prod_{j \in D(u, v)}\left(1-q^{-\tilde{b}_{j}(u, v)}\right) \\
& +q^{l(v)-l(u)-2} \prod_{j \in D(u, v)}\left(1-q^{-\tilde{b}_{j}(u, v)}\right) \\
= & q^{l(v)-l(u)} \prod_{j \in D(u, v)}\left(1-q^{-\tilde{b}_{j}(u, v)}\right)
\end{aligned}
$$

and the result follows. This completes the induction step and hence the proof.

As in the previous section it is natural to rephrase Theorem 4.5 in the language of shifted partitions. Let $\mu, \lambda \in \tilde{\mathcal{I}}(n)$, with $\mu \subseteq \lambda$. We think of $\mu$ and $\lambda$ as paths, as explained at the beginning of this section. Then, by Proposition 4.2 , the path $\lambda$ lies (weakly) above the path $\mu$. Let $j \in[n]$ and consider the $j$-th step of $\lambda$ (from the left). Following [5] we say that such a step is $B$-allowable with respect to $\mu$ if the $j$-th step of $\mu$ is not parallel to it and $\tilde{a}_{j}(\mu, \lambda)$ is odd. For example, if $\mu=(7,4,3,0,0,0,0)$ and $\lambda=(7,6,5,4,2,0,0)$, then the $j$-th step of $\lambda$ is $B$-allowable with respect to $\mu$ exactly if $j \in\{2,5\}$ (see Figure 7).

Proposition 4.6. Let $u, v \in W^{J}, u \leq v$. Then

$$
b_{j}(u, v)=\tilde{a}_{n+1-j}\left(\Lambda_{B}(u), \Lambda_{B}(v)\right)
$$

for $i=1, \ldots, n$. Furthermore $n+1-j \in D(u, v)$ if and only if the $j$-th step of $\Lambda_{B}(v)$ is $B$-allowable with respect to $\Lambda_{B}(u)$.

This result can be proved in a way similar to Proposition 3.5 and is due Brenti [5].

We can now rephrase Theorem 4.5] in terms of shifted partitions.

Corollary 4.7. Let $u, v \in W^{J}, u \leq v$. Then

$$
E_{u, v}(q)=q^{|\lambda \backslash \mu|} \prod_{j}\left(1-q^{-\bar{a}_{j}(\mu, \lambda)}\right),
$$

where $\mu=\Lambda_{B}(u), \lambda=\Lambda_{B}(v), j$ runs over all the $B$-allowable steps of $\lambda$ with respect to $\mu$, and

$$
\bar{a}_{j}(\mu, \lambda):= \begin{cases}2 \tilde{a}_{j}(\mu, \lambda)-1 & \text { if the } j \text {-th step of } \mu \text { is down } \\ 2 \tilde{a}_{j}(\mu, \lambda)+1 & \text { if the } j \text {-th step of } \mu \text { is up. }\end{cases}
$$

In particular, $E_{u, v}(q)$ depends only on $\Lambda_{B}(v) \backslash \Lambda_{B}(u)$. 


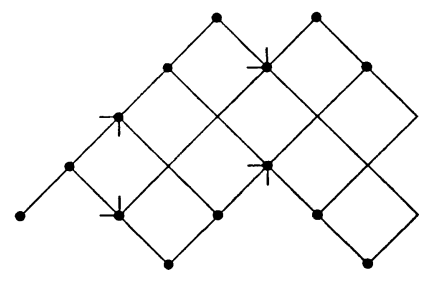

FiguRE 7.

In the case of a lower interval, the formulas (19) and (25) take up a particularly simple form.

Corollary 4.8. Let $v \in W^{J}$. Then

$$
E_{e, v}(q)=q^{l(v)} \prod_{j=1}^{\left\lfloor\frac{N_{1}(v)+1}{2}\right\rfloor}\left(1-q^{-4 j+3}\right)=q^{|\lambda|} \prod_{j=1}^{\left\lfloor\frac{l(\lambda)+1}{2}\right\rfloor}\left(1-q^{-4 j+3}\right),
$$

where $\lambda=\Lambda_{B}(v)$.

Proof. We know from Theorem 4.5 that

$$
E_{e, v}(q)=q^{l(v)} \prod_{j \in D(e, v)}\left(1-q^{-\tilde{b}_{j}(e, v)}\right) .
$$

Clearly, $N(e, v)=\{r \in[n]: v(r)<0\}, \tilde{b}_{j}(e, v)=2 b_{j}(e, v)-1$ for all $j \in[n]$. However, $b_{j}(e, v)=|\{r \geq j: v(r)<0\}|$ for $j \in[n]$, and $D(e, v)=\{r \in N(e, v)$ : $b_{r}(e, v)$ is odd $\}$. Hence,

$$
E_{e, v}(q)=q^{l(v)} \prod_{j=1}^{\left\lfloor\frac{N_{1}(v)+1}{2}\right\rfloor}\left(1-q^{-4 j+3}\right),
$$

as desired. From the definition we have that $l\left(\Lambda_{B}(v)\right)=N_{1}(v)$, so the second equation follows.

We close this section with an example. Let $n=7, u=[4,5,-3,-2,6,7,-1]$, and $v=[6,-5,7,-4,-3,-2,-1] \in\left(S_{7}^{B}\right)^{J}$. We have $N(u, v)=\{2,3,5,6\}$ and $D(u, v)=\{3,6\}$. Since $u(3)<0$ and $u(6)>0$, from (20) and (19) it follows that

$$
E_{u, v}(q)=q^{10}\left(1-q^{-3}\right)\left(1-q^{-1}\right) .
$$

Observe that $\Lambda_{B}(u)=(7,4,3,0,0,0,0)=\mu$ and $\Lambda_{B}(v)=(7,6,5,4,2,0,0)=\lambda$. The paths corresponding to $\Lambda_{B}(v)$ and $\Lambda_{B}(u)$ are drawn in Figure 7 , and the $B$ allowable steps are indicated by arrows. We have that $\bar{a}_{2}(\mu, \lambda)=1$ and $\bar{a}_{5}(\mu, \lambda)=3$.

$$
\text { 5. The CASE }\left(D_{n}, A_{n-1}\right)
$$

In this section we study the $E$-polynomials in the case of the pair $\left(D_{n}, A_{n-1}\right)$. Hence we consider $S_{n}^{D}$, the subgroup of $S_{n}^{B}$ consisting of all the signed permutations having an even number of negative entries in their window notation. More precisely,

$$
S_{n}^{D}:=\left\{w \in S_{n}^{B}: N_{1}(w(1), \ldots, w(n)) \equiv 0(\bmod 2)\right\} .
$$

For the rest of this section, we fix $n \in \mathbf{P}$ and we let $W:=S_{n}^{D}, s_{i}:=(i, i+1)$ $(-i-1,-i)$ for $i=1, \ldots, n-1, s_{0}:=(1,-2)(-1,2), S:=\left\{s_{0}, s_{1}, \ldots, s_{n-1}\right\}$ and 


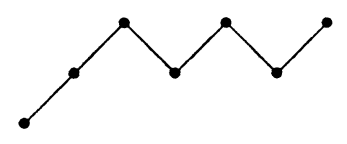

FiguRE 8 .

$J:=S \backslash\left\{s_{0}\right\}$. It is well known that $\left(S_{n}^{D}, S\right)$ is a Coxeter system of type $D_{n}$ and that the following characterization holds (see, e.g., [6]).

Proposition 5.1. Let $v \in S_{n}^{D}$. Then

$$
D(v)=\left\{s_{i} \in S: v(i)>v(i+1)\right\},
$$

where $v(0):=-v(2)$ and $v(n+1):=0$.

As in $\S 4$ we have that $W^{J}=\left\{v \in W: v^{-1}(1)<v^{-1}(2)<\ldots<v^{-1}(n)\right\}$ and for every $v \in W^{J} v \neq e$, there is a unique $k \in[n]$ such that

$$
v^{-1}(k)<0<v^{-1}(k+1)
$$

and we associate to $v$ the shifted partition

$$
\Lambda_{D}(v):=\left(-v^{-1}(1)-1,-v^{-1}(2)-1, \ldots,-v^{-1}(k)-1\right) .
$$

It is not so hard to see that

Proposition 5.2. The map $\Lambda_{D}$ defined by (28) is a bijection between $W^{J}$ and $\tilde{\mathcal{I}}(n-1)$. Furthermore $u \leq v$ in $W^{J}$ if and only if $\Lambda_{D}(u) \subseteq \Lambda_{D}(v)$ and $l(v)=$ $\left|\Lambda_{D}(v)\right|$ for all $u, v \in W^{J}$.

Let $\mathcal{A}$ be the subset of $\tilde{\mathcal{I}}(n)$ consisting of all the shifted partitions with an even number of entries different to zero. More precisely,

$$
\mathcal{A}:=\left\{\lambda=\left(\lambda_{1}, \lambda_{2}, \ldots, \lambda_{k}\right) \in \tilde{\mathcal{I}}(n): k \text { is even }\right\} .
$$

We call this set the even shifted partitions. Observe that $\Lambda_{B}\left(W^{J}\right)=\mathcal{A}$. Since, for $u, v \in W^{J}, u \leq v$ in $\left(S_{n}^{B}\right)^{J}$ if and only if $u \leq v$ in $\left(S_{n}^{D}\right)^{J}$, we have an inclusion preserving bijection between the shifted partition in $\tilde{\mathcal{I}}(n-1)$ and even shifted partitions in $\tilde{\mathcal{I}}(n)$. Thanks to this bijection we can identify $\lambda \in \tilde{\mathcal{I}}(n-1)$ with the lattice path associated to $\lambda \in \tilde{\mathcal{I}}(n)$ as explained in $\S 4$ after Proposition 4.2. We observe that this lattice path starts at $(0,0)$, ends after $n$ steps, and has an even number of up-steps. For example, let $v=[-4,5,-3,6,-2,-1] \in\left(S_{6}^{D}\right)^{J}$. Then $\Lambda_{D}(v)=(5,4,2,0), \Lambda_{B}(v)=(6,5,3,1)$ and the lattice path associate to $v$ is drawn in Figure 8.

Lemma 5.3. Let $v \in W^{J}$ and $j \in[n-1]$. Then $s_{j} \in D(v)$ if and only if $\Lambda_{D}(v)$ has a peak at $n-j$. Furthermore, $s_{o} \in D(v)$ if and only if the last two steps of $\Lambda_{D}(v)$ are up.

Proof. Let $k$ be defined by (27) and $j \in[n-1]$; we have that $s_{j} \in D(v)$ if and only if $v(j)>v(j+1)$. Since $v \in W^{J}$, this happens if and only if $v(j)>0>v(j+1)$. Equivalently, this happens if and only if $j \in v^{-1}([n])$ and $j+1 \notin v^{-1}([n])$. However, $\Lambda_{D}(v)$ (as a path) has a peak at $n-j$ if and only if its $(n-j)$-th step is up and its $(n-j+1)$-th step is down. But the $i$-th step of $\Lambda_{D}(v)$ is an up-step if and only if

$$
i \in\left\{n+1+v^{-1}(1), n+1+v^{-1}(2), \ldots, n+1+v^{-1}(k)\right\} .
$$


Therefore $\Lambda_{D}(v)$ has a peak at $n-j$ if and only if

$$
-j=v^{-1}(i)+1
$$

for some $i \in[k]$, and

$$
-j \neq v^{-1}(i)
$$

for all $i \in[k]$. Equivalently, if and only if $j \notin v^{-1}([-k,-1])$ and $j+1 \in v^{-1}([-k,-1])$, but by the definition of $k, i \notin v^{-1}([-k,-1])$ if and only if $i \notin v^{-1}([-n,-1])$, which is if and only if $i \in v^{-1}([n])$, for all $i \in[n]$. The result follows.

Now let $s_{o} \in D(v)$. This happens if and only if $v(1)+v(2)<0$ and implies that $v(1)<v(2)<0$. It follows that $v^{-1}(k)=-1$ and $v^{-1}(k-1)=-2$, and that the $n$-th and the $n-1$-th steps of $\Lambda_{D}(v)$ are up.

The following is exactly the analogue of Proposition 4.4 for permutation in $\left(S_{n}^{D}\right)^{J}$.

Proposition 5.4. Let $v \in W^{J}$ and $i \in[n]$. Then the $i$-th step (from the left) of $\Lambda_{D}(v)$ (seen as a path) is an up-step if and only if $v(n+1-i)<0$.

Now we are ready to prove the analogue of Theorem 4.5 for the permutations in $\left(S_{n}^{D}\right)^{J}$. The formula is exactly the same, but observe that the polynomials are not always the same; in fact the function length is different.

Theorem 5.5. Let $u, v \in W^{J}, u \leq v$. Then

$$
E_{u, v}(q)=q^{l(v)-l(u)} \prod_{j \in D(u, v)}\left(1-q^{-\tilde{b}_{j}(u, v)}\right),
$$

where $\tilde{b}_{j}(u, v)$ is defined as in Theorem 4.5 .

Proof. We proceed by induction on $l\left(w_{0}^{J}\right)-l(u)$. If $l\left(w_{0}^{J}\right)-l(u)=0$, we have $w_{0}^{J}=v=u$ and the result is trivially true. So suppose that $l\left(w_{0}^{J}\right)-l(u)>0$ and let $s$ be such that $s \notin D(u)$ and $u s \in W^{J}$. If $s=(-i-1,-i)(i, i+1)$ for some $i \in[n-1]$, then the proof is exactly the same as for Theorem 4.5. So suppose that $s=(-1,2)(1,-2)=s_{0}$; then we have $u(1)+u(2)>0$. We observe that $u s \in W^{J}$ implies that $0<u(1)<u(2)$. Moreover we have that $b_{1}(u, v)$ is even for every $u, v \in W^{J}$, and so $1 \notin D(u, v)$. We have four cases to consider.

a) $s \notin D(v)$ and $v s \in W^{J}$.

We have $0<v(1)<v(2)$. Then $N(u s, v s)=N(u, v)$ and $b_{j}(u s, v s)=b_{j}(u, v)$ for all $j \in[n]$ and so $D(u s, v s)=D(u, v)$ and $\tilde{b}_{j}(u s, v s)=\tilde{b}_{j}(u, v)$ for all $j \in[n] \backslash\{1,2\}$. Hence, by Theorem 2.1 and our induction hypothesis,

$$
\begin{aligned}
E_{u, v}(q) & =E_{u s, v s}(q) \\
& =q^{l(v)-l(u)} \prod_{j \in D(u, v)}\left(1-q^{-\tilde{b}_{j}(u, v)}\right) .
\end{aligned}
$$

b) $s \in D(v)$ and $u s \not \leq v s$.

Hence $v(1)+v(2)<0$ and $v(1)<v(2)<0$. We have that $N(u s, v)=N(u, v) \backslash\{1,2\}$, $b_{j}(u s, v)=b_{j}(u, v)$ for all $j \in[n] \backslash\{1,2\}$. Using reasoning as in Theorem 4.5 b), $b_{1}(u, v)>b_{2}(u, v) \geq 0$, so $b_{1}(u, v) \geq 2 ; b_{2}(u s, v s)>b_{1}(u s, v s)$ so $b_{1}(u s, v s) \leq-2$, and $b_{1}(u, v)-b_{1}(u s, v s)=4$ so $b_{1}(u, v)=2$ and $b_{2}(u, v)=1$. So $D(u s, v)=$ 
$D(u, v) \backslash\{2\}$ and $\tilde{b}_{j}(u s, v)=\tilde{b}_{j}(u, v)$ for all $j \in[n] \backslash\{1,2\}$ and $\tilde{b}_{2}(u, v)=1$. Hence, by Theorem 2.1 and the induction hypothesis,

$$
\begin{aligned}
E_{u, v}(q) & =(q-1) E_{u s, v}(q) \\
& =(q-1) q^{l(v)-l(u)-1} \prod_{j \in D(u, v) \backslash\{2\}}\left(1-q^{-\tilde{b}_{j}(u, v)}\right) \\
& =q^{l(v)-l(u)} \prod_{j \in D(u, v)}\left(1-q^{-\tilde{b}_{j}(u, v)}\right) .
\end{aligned}
$$

c) $s \in D(v)$ and $u s \leq v s$.

We have $v(1)+v(2)<0$ and $v(1)<v(2)<0$. So $N(u s, v s)=N(u, v), N(u s, v)=$ $N(u, v) \backslash\{1,2\}, b_{j}(u s, v s)=b_{j}(u, v)=b_{j}(u s, v)$ for all $j \in[n] \backslash\{1,2\}$, and $b_{2}(u s, v s)=b_{2}(u, v)-2$. It follows that

$$
D(u s, v)=D(u, v) \backslash\{2\}, \quad D(u s, v s)=D(u, v),
$$

$\tilde{b}_{j}(u s, v s)=\tilde{b}_{j}(u, v)=\tilde{b}_{j}(u s, v)$ for all $j \in[n] \backslash\{1,2\}$, and $\tilde{b}_{2}(u s, v s)=\tilde{b}_{2}(u, v)-2$. Hence, by Theorem 2.1 and our induction hypothesis,

$$
\begin{aligned}
E_{u, v}(q) & =\left(q-q^{-1}\right) E_{u s, v}(q)+E_{u s, v s}(q) \\
& =\left(q-q^{-1}\right) q^{l(v)-l(u)-1} \prod_{j \in D(u, v) \backslash\{2\}}\left(1-q^{-\tilde{b}_{j}(u, v)}\right) \\
& +q^{l(v)-l(u)-2}\left(1-q^{-\tilde{b}_{2}(u s, v s)}\right) \prod_{j \in D(u, v) \backslash\{2\}}\left(1-q^{-\tilde{b}_{j}(u, v)}\right) \\
& =q^{l(v)-l(u)} \frac{\left(1-q^{-\tilde{b}_{2}(u s, v s)-2}\right)}{\left(1-q^{-\tilde{b}_{2}(u, v)}\right)} \prod_{j \in D(u, v)}\left(1-q^{-\tilde{b}_{j}(u, v)}\right),
\end{aligned}
$$

and the result follows.

d) $v s \notin W^{J}$.

We have two cases. Suppose that $v(1)>0$ and $v(2)<0$. We have $N(u s, v)=$ $(N(u, v) \cup\{1\}) \backslash\{2\}, b_{j}(u, v)=b_{j}(u s, v)$ for all $j \in[n] \backslash\{1,2\}$, and

$$
\begin{gathered}
b_{1}(u, v)=b_{2}(u, v), \\
b_{1}(u s, v)=b_{1}(u, v)-2 .
\end{gathered}
$$

Since $b_{1}(u, v)$ is even, (33) implies that $2 \notin D(u, v)$, and (34) implies that $1 \notin$ $D(u s, v)$. It follows that $D(u, v)=D(u s, v)$ and $\tilde{b}_{j}(u s, v)=\tilde{b}_{j}(u, v)$ for all $j \in$ $[n] \backslash\{1,2\}$, so the thesis follows immediately by induction.

Suppose now that $v(1)<0$ and $v(2)>0$. Then $N(u s, v)=(N(u, v) \cup\{2\}) \backslash\{1\}$, $b_{j}(u, v)=b_{j}(u s, v)$ for all $[n] \backslash\{1,2\}$, and

$$
b_{1}(u, v)=b_{2}(u s, v)+2 .
$$

Since $b_{1}(u, v)$ is even, (35) implies that $2 \notin D(u s, v)$. It follows that $D(u, v)=$ $D(u s, v)$ and $\tilde{b}_{j}(u s, v)=\tilde{b}_{j}(u, v)$ for all $j \in[n] \backslash\{1,2\}$, so the thesis follows immediately by induction. This completes the proof.

As in the previous cases it is natural to rephrase Theorem [5.5 in the language of shifted partitions. Let $\lambda, \mu \in \mathcal{A}$, with $\lambda \subseteq \mu$. We think of $\mu$ and $\lambda$ as paths as explained in $\S 4$. So using Proposition 4.6] we have that 
Corollary 5.6. Let $u, v \in W^{J}, u \leq v$. Then

$$
E_{u, v}(q)=q^{|\lambda \backslash \mu|} \prod_{j}\left(1-q^{-\bar{a}_{j}(\mu, \lambda)}\right)
$$

where $\mu=\Lambda_{D}(u), \lambda=\Lambda_{D}(v), j$ runs over all the B-allowable steps of $\lambda$ with respect to $\mu$, and $\bar{a}_{j}(u, v)$ is defined as in Corollary 4.7. In particular, $E_{u, v}(q)$ depends only on $\Lambda_{D}(v) \backslash \Lambda_{D}(u)$.

For lower intervals we obtain the following

Corollary 5.7. Let $v \in W^{J}$. Then

$$
E_{e, v}(q)=q^{l(v)} \prod_{j=1}^{\frac{N_{1}(v)}{2}}\left(1-q^{-4 j+3}\right)=q^{|\lambda|} \prod_{j=1}^{\frac{l(\lambda)}{2}}\left(1-q^{-4 j+3}\right),
$$

where $\lambda=\Lambda_{D}(v)$.

Proof. The results follows immediately from Corollary 4.8 , observing that $N_{1}(v)$ is even for every $v \in W^{J}$.

\section{The Cases $\left(B_{n}, B_{n-1}\right)$ And $\left(D_{n}, D_{n-1}\right)$}

In this section we analyze the $E$-polynomials in the cases $\left(B_{n}, B_{n-1}\right)$ and $\left(D_{n}, D_{n-1}\right)$. We start with the first one. We use the same notations as in $\S 4$, hence $W=S_{n}^{B}, S=\left\{s_{0}, s_{1}, \ldots, s_{n-1}\right\}$, and $s_{o}=(-1,1)$, but now we let $J:=S \backslash\left\{s_{n-1}\right\}$, so as to have $W_{J}=B_{n-1}$. It follows that the quotient $W^{J}$ is a totally ordered set, more precisely, is the chain

$$
\begin{aligned}
W^{J}=\left\{e, s_{n-1}, \ldots, s_{n-1} s_{n-2} \ldots s_{1} s_{0}, s_{n-1} \ldots s_{1} s_{0} s_{1}, \ldots,\right. \\
\left.s_{n-1} s_{n-2} \ldots s_{1} s_{0} s_{1} \ldots s_{n-1}\right\} .
\end{aligned}
$$

Proposition 6.1. Let $(W, S)$ be a Coxeter system, $J \subset S$ and $u, v \in W^{J}$ such that $u \leq v$. If $[u, v]^{J}$ is a chain, then

$$
E_{u, v}(q)=q^{l(v)-l(u)}\left(1-q^{-1}\right) .
$$

Proof. We proceed by induction on $l\left(w_{0}^{J}\right)-l(u)$. If $l\left(w_{0}^{J}\right)-l(u)=0$, the result is trivially true. So suppose that $l\left(w_{0}^{J}\right)-l(u)>0$ and let $s \notin D(u)$ and $u s \in W^{J}$. We have four cases to consider.

a) $s \notin D(v)$ and $v s \in W^{J}$.

Then the result follows immediately by induction.

b) $s \in D(v)$ and $u s \not \leq v s$.

We know that $[u, v]^{J}$ is a chain, so us $\not \leq v s$ implies that $u s=v$. It follows that

$$
E_{u, v}(q)=(q-1) E_{u s, v}(q)=q\left(1-q^{-1}\right) .
$$

c) $s \in D(v)$ and $u s \leq v s$.

We have

$$
\begin{aligned}
E_{u, v}(q) & =\left(q-q^{-1}\right) q^{l(v)-l(u)-1}\left(1-q^{-1}\right)+q^{l(v)-l(u)-2}\left(1-q^{-1}\right) \\
& =q^{l(v)-l(u)-2}\left(1-q^{-1}\right) q^{2}=q^{l(v)-l(u)}\left(1-q^{-1}\right) .
\end{aligned}
$$




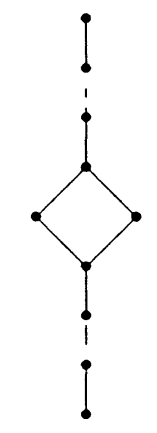

FiguRE 9.

d) $v s \notin W^{J}$.

Then the result follows immediately by induction.

By the comment preceding the proposition this settles the case $\left(B_{n}, B_{n-1}\right)$.

Let us examine the case $\left(D_{n}, D_{n-1}\right)$. Using the notations of $\S 5$ we let $W=D_{n}$, $S=\left\{s_{0}, \ldots, s_{n-1}\right\}$, and $s_{0}=(-1,2)(1,-2)$. Now we let $J:=S \backslash\left\{s_{n-1}\right\}$. The quotient can be written in the form

$$
W^{J}=\left\{w \in W: w^{-1}(-2)<w^{-1}(1)<\ldots<w^{-1}(n-1)\right\},
$$

and its Bruhat order is drawn in Figure 9.

Moreover we know the unique reduced expression of each $w \in W^{J}$, in fact the $n-1$ elements in the chain on the bottom are $e, s_{n-1}, \ldots, s_{n-1} \cdots s_{2}$, the $n-1$ elements in the chain on the top are $s_{n-1} \cdots s_{2} s_{0} s_{1}, \ldots, s_{n-1} \cdots s_{2} s_{0} s_{1} s_{2} \cdots s_{n-1}$, and the remaining two elements are exactly $s_{n-1} \cdots s_{2} s_{0}$ and $s_{n-1} \cdots s_{2} s_{1}$. So every $i \in[2, n-1]$ identifies two elements in the quotient, $u_{i}:=s_{n-1} \cdots s_{i}$ in the chain on the bottom, and $v_{i}:=s_{n-1} \cdots s_{2} s_{0} s_{1} \cdots s_{i}$ in the chain on the top. Moreover, we define $u_{n}:=e$.

Note that for all $i \in[2, n]$ and for all $j \in[1, n-1]$ we have $l_{D}\left(u_{i}\right)=n-i$ and $l_{D}\left(v_{j}\right)=n+j-1$.

We call $(u, v)$ a equidistant pair if there exists $i \in[2, n]$ such that $u=u_{i}$ and $v=v_{i-1}$.

The verification of the following observation is left to the reader.

Lemma 6.2. Let $u, v \in W^{J}$. If $(u, v)$ is an equidistant pair, then $u^{-1}(j)=v^{-1}(j)$ for all $j \in[2, n-1]$.

Proposition 6.3. Let $u, v \in W^{J}$. Then

$$
E_{u, v}(q)= \begin{cases}\left(q^{l(v)-l(u)}-q\right)\left(1-q^{-1}\right) & \text { if }(u, v) \text { is equidistant } \\ q^{l(v)-l(u)}\left(1-q^{-1}\right) & \text { otherwise. }\end{cases}
$$

Proof. We have three cases to consider.

1) $(u, v)=\left(u_{i}, v_{j}\right)$, with $i \in[2, n]$ and $j \in[1, n-1] \backslash\{i-1\}$.

If $j \geq i$, then $u_{i}=s_{n-1} \cdots s_{i}$ and $v_{j}=s_{n-1} \cdots s_{2} s_{0} s_{1} \cdots s_{j}$. Hence $s_{i-1} \notin D\left(u_{i}\right)$ and $u_{i} s_{i-1}=u_{i-1} \in W^{J}$, while $v_{j} s_{i-1} \notin W^{J}$, so we have $E_{u_{i}, v_{j}}(q)=q E_{u_{i-1}, v_{j}}(q)$. We have $i-2$ steps like this to do, and one more step for $s=s_{0}$, so at the end we 
have

$$
E_{u_{i}, v_{j}}(q)=q^{i-2} E_{u_{2}, v_{j}}(q)=q^{i-1} E_{u_{2} s_{0}, v_{j}}(q) .
$$

Now $\left[u_{2} s_{0}, v_{j}\right]^{J}$ is a chain with $j$ steps and so by Proposition 6.1 it follows that $E_{u_{2} s_{0}, v_{j}}(q)=q^{j-1}(q-1)$ and so from (37) we are done.

If $j<i-1$, after $(i-j-2)$ steps in the diagram we have

$$
E_{u_{i}, v_{j}}(q)=q^{i-j-2} E_{u_{j+2}, v_{j}}(q) \text {. }
$$

The next step is for $s=s_{j+1} ; s_{j+1} \notin D\left(u_{j+2}\right)$ and $s_{j+1} \notin D\left(v_{j}\right)$, so by the first part of the proof we have

$$
E_{u_{j+2}, v_{j}}(q)=E_{u_{j+1}, v_{j+1}}(q)=q^{2 j+1}\left(1-q^{-1}\right),
$$

and the result follows from (38).

2) $(u, v)$ is an equidistant pair.

We proceed by induction on $i$. If $i=2$, then $u_{2}=s_{n-1} \cdots s_{2}$ and $v_{1}=s_{n-1} \cdots s_{2} s_{0} s_{1}$. Hence $s_{0} \notin D\left(u_{2}\right), s_{0} \in D\left(v_{1}\right)$ and $u_{2} s_{0} \not \leq v_{1} s_{0}$, so $E_{u_{2}, v_{1}}(q)=(q-1) E_{u_{2} s_{0}, v_{1}}(q)$. In the second step, $s:=s_{1}$, we have $u_{2} s_{0} s_{1} \not \leq v_{1} s_{1}$, so $E_{u_{2}, v_{1}}(q)=(q-1)^{2} E_{u_{2} s_{0} s_{1}, v_{1}}(q)$ $=(q-1)^{2}$, since $v_{1}=u_{2} s_{0} s_{1}$.

So suppose $i>2$. Then $s_{i-1} \notin D\left(u_{i}\right), u_{i} s_{i-1}=u_{i-1} \in W^{J}$, and $v_{i-1} s_{i-1}=$ $v_{i-2} \in W^{J}$, so since $u_{i-1} \leq v_{i-2}$, we have

$$
E_{u_{i}, v_{i-1}}(q)=\left(q-q^{-1}\right) E_{u_{i-1}, v_{i-1}}(q)+E_{u_{i-1}, v_{i-2}}(q) .
$$

By case 1), $E_{u_{i-1}, v_{i-1}}(q)=q^{l\left(v_{i-1}\right)-l\left(u_{i-1}\right)}\left(1-q^{-1}\right)$ and by induction, $E_{u_{i-1}, v_{i-2}}(q)$ $=q^{l\left(v_{i-2}\right)-l\left(u_{i-1}\right)}\left(1-q^{-1}\right)$. The result follows.

3) $[u, v]^{J}$ is a chain.

The result follows by Proposition 6.1 This completes the proof.

\section{Consequences And Further Remarks}

In this section we derive some consequences of our results. We start by proving that the E-polynomials are combinatorial invariants, i.e. that they depend only on the poset $[u, v]^{J}$. To do this we need a purely order theoretic result on skew partitions that was first proved in [4, Lemma 5.5].

Lemma 7.1. Let $\rho, \nu$ be two connected skew partitions that are isomorphic as posets. Then either $\rho \approx \nu$ or $\rho \approx \nu^{\prime}$.

We can now prove the main result of this section.

Corollary 7.2. Let $J \subset S$, as in $\S 3$, $\S 4$, and $\S 5$, and $u, v \in W^{J}, x, y \in W^{J}$ be such that $[u, v]^{J} \cong[x, y]^{J}$. Then

$$
E_{u, v}(q)=E_{x, y}(q) .
$$

Proof. Now we prove this result in the case when $W=S_{n}$. By Proposition 3.2 we have that $[u, v]^{J}$ is isomorphic, as a poset, to the interval $[\Lambda(u), \Lambda(v)]$ in Young's lattice. But it follows immediately from the definitions and well-known results in the theory of partially ordered sets (see, e.g., [18, §3.4]) that the subposet of joinirreducibles of $[\Lambda(u), \Lambda(v)]$ is isomorphic to $\Lambda(v) \backslash \Lambda(u)$, where the skew partition $\Lambda(v) \backslash \Lambda(u)$ is seen as a poset. Therefore, since $[u, v]^{J} \cong[x, y]^{J}$, we conclude that $\Lambda(v) \backslash \Lambda(u) \cong \Lambda(y) \backslash \Lambda(x)$ (as poset), and the result follows from Lemma 7.1 and Corollary 3.6 . 
Similarly, we can prove the result for the other cases, but we need to replace Proposition 3.2 Corollary [3.6 and $\Lambda$ for $\left(C_{n}, A_{n-1}\right)$ with Proposition 4.2, Corollary [4.7 and $\Lambda_{B}$, and for $\left(D_{n}, A_{n-1}\right)$ with Proposition [5.2, Corollary [5.6] and $\Lambda_{D}$, respectively.

Note that in the case when $W=S_{n}$ the proof of Corollary 7.2 applies whenever $[u, v]^{K} \cong[x, y]^{H}$ with $K, H \subset S,|K|=|H|=|S|-1$.

We conclude this section by discussing the connections mentioned at the end of the Introduction. In [14], Gabber and Joseph define for every $u, v \in W$ a polynomial

$$
R_{u, v}^{\prime}(q)=\sum_{k \geq 0}(-1)^{l(v)-l(u)-k} q^{k} \operatorname{dim}\left(\operatorname{Ext}^{k}\left(M_{u}, M_{v}\right)\right)
$$

and they conjectured (although this is not explicitly stated) that

$$
R_{u, v}^{\prime}(q)=R_{u, v}(q)
$$

This conjecture is not true (see [2]), but the $R^{\prime}$ and the $R$-polynomials are not so different. In fact, Carlin shows that the $R^{\prime}$-polynomials are monic of degree $l(v)-l(u)$ (see [8. Theorem 3.8]), as are the $R$-polynomials, and proves that the conjecture is true in two cases: when $l(v)-l(u) \leq 3$ (see [8, Proposition 3.13]) and when $(u, v)$ is a Coxeter pair ( 8 , Proposition 3.11]). The $E$-polynomials play the same role as the $R^{\prime}$-polynomials in the generalized case, so it is natural to wonder about the analogous question, i.e. if $E_{u, v}(q)=R_{u, v}^{J}(q)$, where $R_{u, v}^{J}(q)$ are the parabolic $R$-polynomials (see e.g., [12]). This question also has a negative answer. In fact, for example, let $v=[3,4,1,2,5] \in S_{5}^{S \backslash\{(2,3)\}}$. Then we have that $R_{e, v}^{J}(q)=q^{4}\left(1-q^{-1}\right)\left(1-q^{-2}\right)$, while $E_{e, v}(q)=q^{4}\left(1-q^{-1}\right)\left(1-q^{-3}\right)$.

However, we can prove the analogue of the results of Carlin, for generalized Verma modules. The first two results are very simple, and their proofs are immediate from Theorems $3.4,4.5$ and 5.5 ,

Corollary 7.3. Let $u, v \in W^{J}, u \leq v$. Then $E_{u, v}(q)$ is a monic polynomial of degree $l(v)-l(u)$.

Corollary 7.4. Let $u, v \in W^{J}$. If $u \leq v$, then

$$
\operatorname{dim}\left(\operatorname{Ext}^{l(v)-l(u)}\left(N_{u}, N_{v}\right)\right)=1 .
$$

In [4] Brenti finds explicit formulas for the maximal parabolic $R$-polynomials of the symmetric group and, in [5] for the group of signed permutations, when $J=S \backslash\left\{s_{0}\right\}$. He proves the following

Theorem 7.5. Let $u, v \in S_{n}^{J}, u \leq v$. Then

$$
R_{u, v}^{J}(q)=q^{|\Lambda(v) \backslash \Lambda(u)|} \prod_{j}\left(1-q^{-\tilde{a}_{j}(\Lambda(u), \Lambda(v))}\right)
$$

where $j$ runs over the allowable steps of $\Lambda(v)$ with respect to $\Lambda(u)$.

Theorem 7.6. Let $u, v \in\left(S_{n}^{B}\right)^{J}, u \leq v$. Then

$$
R_{u, v}^{J}(q)=q^{\left|\Lambda_{B}(v) \backslash \Lambda_{B}(u)\right|} \prod_{j}\left(1-q^{-\bar{b}_{j}\left(\Lambda_{B}(u), \Lambda_{B}(v)\right)}\right)
$$


where $j$ runs over the $B$-allowable steps of $\Lambda_{B}(v)$ with respect to $\Lambda_{B}(u)$ and $\bar{b}_{j}\left(\Lambda_{B}(u), \Lambda_{B}(v)\right):= \begin{cases}\tilde{a}_{j}\left(\Lambda_{B}(u), \Lambda_{B}(v)\right) & \text { if the } j \text {-th step of } \Lambda_{B}(u) \text { is down, } \\ \tilde{a}_{j}\left(\Lambda_{B}(u), \Lambda_{B}(v)\right)+1 & \text { if the } j \text {-th step of } \Lambda_{B}(u) \text { is up. }\end{cases}$

The author will prove in another paper that the formula in Theorem 7.6 can be extended to the case $W=S_{n}^{D}$, but we need to replace $\Lambda_{B}$ by $\Lambda_{D}$.

Our formulas are very similar to Brenti's, and it is easy to see that the following results hold. The statements and proofs are given for $W=S_{n}$, but the results are also true for $S_{n}^{B}$ and $S_{n}^{D}$. We simply need to replace $\Lambda$ by $\Lambda_{B}$ and $\Lambda_{D}$, respectively.

We say that a skew partition is a border strip (also called a ribbon) if it contains no $2 \times 2$ square of cells.

Proposition 7.7. Let $u, v \in W^{J}$ be such that the skew partition $\Lambda(v) \backslash \Lambda(u)$ is a border strip. Then

$$
E_{u, v}(q)=R_{u, v}^{J}(q)
$$

So, in particular, we obtain the analogue of Proposition 3.13 of 8 .

Corollary 7.8. Let $u, v \in W^{J}$. If $l(v)-l(u) \leq 3$, then

$$
\operatorname{dim}\left(\operatorname{Ext}^{k}\left(N_{u}, N_{v}\right)\right)=r_{k}^{J}(u, v),
$$

where $r_{k}^{J}(u, v)$ is the absolute value of the coefficient of $q^{k}$ in $R_{u, v}^{J}(q)$.

Let $u, v \in W^{J}$; we call $(u, v)$ a generalized Coxeter pair if $r_{1}^{J}(u, v)=l(v)-l(u)$. The next result is the analogue of Proposition 3.11 of 8 .

Proposition 7.9. Let $u, v \in W^{J}$. If $(u, v)$ is a generalized Coxeter pair, then

$$
\operatorname{dim}\left(\operatorname{Ext}^{k}\left(N_{u}, N_{v}\right)\right)=r_{k}^{J}(u, v)=\left(\begin{array}{l}
n \\
k
\end{array}\right)
$$

for $k=0, \ldots, n$, where $n=l(v)-l(u)$.

Proof. If $(u, v)$ is a generalized Coxeter pair, then the only possibility is that $R_{u, v}^{J}(q)$ $=q^{n}\left(1-q^{-1}\right)^{n}$. This means that the skew partition $\Lambda(v) \backslash \Lambda(u)$ is a border strip, and so the result follows by Proposition 7.7 .

It seems that the situation is exactly analogous, hence we are led to think that, as for the generalized Verma modules, there should also exist a recursion formula for the ordinary ones. So, we close this paper with this proposal.

Problem. Find a recursion formula for the $R^{\prime}$-polynomials.

\section{ACKNOWLEDGEMENTS}

I am very grateful to my advisor, Francesco Brenti, for suggesting the problem to me and especially for his generous support. I would like to thank David Vogan and George Lusztig for some useful conversations, and M.I.T. for its hospitality during the preparation of this work. 


\section{REFERENCES}

[1] I.N. Bernstein, I.M. Gelfand, S.I. Gelfand, On a category of g-modules, Funct. Anal. Appl., 10 (1976), 1-8. MR 53:10880

[2] B. Boe, A Counterexample to the Gabber-Joseph Conjecture, Kazhdan-Lusztig Theory and related topics, Contemp. Math., 139, 1-2, Amer. Math. Soc., Providence, RI, 1992. MR 94a:22032

[3] B. Boe, T.J. Enright, B. Shelton, Determination of the interwining operators for holomorphically induced representations of Hermitian symmetric pairs, Pacific J. Math., 131 (1988), 39-50. MR 88m:22038

[4] F. Brenti, Kazhdan-Lusztig and R-polynomials, Young's lattice, and Dyck partitions, Pacific J. Math., to appear.

[5] F. Brenti, personal communication, December 2000.

[6] F. Brenti, q-Eulerian Polynomials Arising from Coxeter Groups, Europ. J. Combinatorics, 15 (1994), 417-441. MR 95i:05013

[7] J. Brylinski, M. Kashiwara, Kazhdan-Lusztig conjecture and holonomic systems, Invent. Math., 64 (1981), 387-410. MR 83e:22020

[8] K.J. Carlin, Extensions of Verma Modules, Trans. Amer. Math. Soc., 294 (1986), 29-43. MR 87j:17006

[9] L. Casian, D. Collingwood, The Kazhdan-Lusztig conjecture for generalized Verma modules, Math. Z., 195 (1987), 581-600. MR 88i:17008

[10] D.H. Collingwood, The n-Homology of Harish-Chandra Modules: Generalizing a theorem of Kostant, Math. Ann., 272 (1985), 161-187. MR 87a:22027

[11] D.H. Collingwood, B. Shelton, A duality theorem for extensions on induced Highest Weight modules, Pacific. J. Math., 146 (1990), 227-237. MR 91m:22029

[12] V. Deodhar, On some geometric aspects of Bruhat orderings. II. The parabolic analogue of Kazhdan-Lusztig polynomials, J. Algebra, 111 (1987), 483-506. MR 89a:20054

[13] T.J. Enright, B. Shelton, Categories of highest weight modules: applications to Hermitian symmetric spaces, Mem. Amer. Math. Soc., 367 (1987). MR 88f:22052

[14] O. Gabber, A. Joseph, Towards the Kazdhan-Lusztig conjecture, Ann. Sci. École. Norm. Sup., 14 (1981), 261-302. MR 83e:17009

[15] J. E. Humphreys, Reflection Groups and Coxeter Groups, Cambridge Stud. Adv. Math., no. 29, Cambridge Univ. Press, Cambridge, 1990. MR 92h:20002

[16] D. Kazhdan, G. Lusztig, Representations of Coxeter groups and Hecke algebras, Invent. Math., 53 (1979), 165-184. MR 81j:20066

[17] B. Shelton, Extensions Between Generalized Verma Modules: The Hermitian Symmetric Case, Math. Z., 197 (1988), 305-318. MR 89d:17006

[18] R. P. Stanley, Enumerative Combinatorics, vol. 1, Wadsworth and Brooks/Cole, Monterey, CA, 1986. MR 87j:05003

[19] R. P. Stanley, Enumerative Combinatorics, vol. 2, Cambridge Stud. Adv. Math., no. 62, Cambridge Univ. Press, Cambridge, 1999. MR 2000k:05026

Dipartimento di Matematica, Università di Roma "La Sapienza", 00185 Roma, Italy Current address: LACIM, Université du Quebéc à Montréal, case postale 8888, succursale Centre-Ville, Montréal, Quebéc, Canada H3C 3P8

E-mail address: biagioli@math.uqam.ca 